\title{
EXPERIMENTACIÓN Y PROPIEDADES COMBUSTIBLES DE ESPECIES DEL BOSQUE ANDINO PATAGÓNICO APORTACIÓN AL ESTUDIO ANTRACOLÓGICO DE SITIOS ARQUEOLÓGICOS
}

LAURA CARUSO F.

\begin{abstract}
RESUMEN
Conocer las propiedades combustibles de las especies leñosas permite complementar el análisis e interpretación de las modalidades de adquisición y uso del material leñoso por parte de grupos del pasado. El método experimental permite profundizar en las propiedades que las distintas especies vegetales poseen como combustible, pero más importante aun posibilita la reconstrucción de los procesos que han generado el conjunto de restos recuperados. Este trabajo presenta dos objetivos. Por un lado determinar las cualidades que poseen como combustible cuatro especies leñosas (austrocedrus chilensis, fitzroya cupressoides, nothofagus antarctica y nothofagus dombeyi) y comprender su comportamiento en el proceso de combustión. Por otro lado, explorar si las grietas de contracción presentes en los carbones responden al estado de la madera puesta al fuego (verde-seco). La realización de 16 fuegos experimentales y el estudio de sus carbones evidencian diferencias entre las especies leñosas estudiadas. Los resultados obtenidos permiten por un lado, discriminar la utilización de madera seca y madera verde como combustible. Por otro, profundizar en la identificación e interpretación de las alteraciones presentes en los carbones arqueológicos y complementar el análisis arqueobotánico de los distintos sitios patagónicos.
\end{abstract}

PALABRAS CLAVE: experimentación, propiedades combustibles, bosque andino-patagónico.

\section{EXPERIMENTATION AND COMBUSTION PROPERTIES OF PATAGONIAN ANDEAN FOREST. CONTRIBUTION TO THE ARCHAEOBOTANICAL STUDIES OF ARCHAEOLOGICAL SITES}

\begin{abstract}
The properties of woody fuels during the combustion can complement the interpretation of the modes of acquisition and use of firewood by groups of the past. The experimental method allows to deepen about the properties of different plant species as fuel, but more importantly allows the reconstruction of the processes that have generated the archaeobotanical remains recovered. This paper has two objectives. For one determine the combustion properties of four woody species (austrocedrus, fitzroya cupressoides, nothofagus and nothofagus antarctica dombeyi) and understand their behavior in the combustion process. Furthermore, explore whether shrinkage cracks present in the charcoal respond to

\footnotetext{
* Laboratori d'Arqueobotànica, Departament de Prehistòria. Universitat Autònoma de Barcelona (unitat asociada al CSIC-Institución Milá y Fontanals). AGREST research GROUP. lcarusoferme@gmail.com.
} 
water content the firewood (green-dry). The performance of 16 experimental combustions and the study of the charcoal produced during combustion show differences between woody species studied. The results allow, in some cases, to discriminate the use of dry wood and green wood as fuel. The results obtained allow on one hand, discriminate the use of dry wood and green wood as fuel. On the other, deepen the identification and interpretation of the alterations present in the archaeological charcoals and complement the archaeobotanical analysis of the different sites patagonia.

KEY WORDS: experimentation, combustion properties, andean patagonian forest.

\section{INTRODUCCIÓN}

Desde la década de los años 70' el uso del fuego, por parte de los grupos del pasado, es objeto de estudio en distintos tipos de investigaciones. Existen trabajos orientados al estudio de las técnicas de producción del fuego (Collina Girard 1993, Perlès 1977), al análisis de las estructuras de combustión (March 1996, March et al. 1989, Orliac \& Wattez 1989) y al estudio de sus residuos (Chabal 1992, Chabal et al., 1999, Théry-Parisot, 1998, 2001). No obstante, en pocas ocasiones la conservación de restos vegetales permite llevar a cabo un estudio integral de los varios tipos de recursos vegetales utilizados, así como de las diferentes modalidades de procesado y consumo del material leñoso (Caruso et al., 2008). Los restos vegetales presentes en los sitios arqueológicos corresponden a aquellas partes más resistentes a la descomposición, o a aquellos que se han depositado en un contexto favorable para su conservación (Hastorf, 1999, Buxó 1997, Buxó \& Piqué, 2003, entre otros). Por lo tanto, para comprender el comportamiento de las especies leñosas en el proceso de combustión y determinar sus cualidades como combustible es necesario el desarrollo de un trabajo experimental (Bazile-Robert, 1982, Théry-Parisot 1998, Thèry-Parisot \& Costamagno, 2005). La experimentación no solo permitirá conocer las propiedades que las distintas especies poseen como combustible, sino que también posibilitará la reconstrucción de los procesos que han generado el conjunto de restos recuperados. Por ello, el método experimental complementa el análisis e interpretación de las modalidades de adquisición y uso del material leñoso por parte de grupos del pasado.

En arqueobotánica la experimentación es utilizada con diferentes objetivos, como por ejemplo comprender la naturaleza de los distintos tipos de alteraciones presentes en los carbones, responder interrogantes relacionados con el proceso de combustión, etc. (Allué et al., 2007; Andreoni et al., 2011, Braadbaa et al., 2009, Caruso, 2008, 2010, 2012; Caruso \& Thèry -Parisot, 2011; Dufraisse et al., 2010, Mcparland et al., 2010, 2006 , etc.). Sin embargo, cabe tener presente que toda experimentación presenta ciertas limitaciones generadas por la dificultad de reproducir de manera idéntica las situaciones pasadas.

Por lo que se refiere al uso del fuego entre grupos cazadores-recolectores patagónicos, en general los trabajos realizados se centran en el estudio de restos de madera carbonizada (Marconetto, 2002, Ortega \& Marconetto, 2011, Pérez de Micou, 1988, 1999, Piqué, 1999, Rodríguez, 2003-2005, Solari 1993, 2009, entre otros), siendo escasa por lo tanto la información sobre las propiedades combustibles de las distintas especies vegetales. Dada la escasez e importancia de dicha información en el estudio de la gestión de la materia prima leñosa por un lado, y el estudio arqueobotánico llevado a cabo en el sitio Paredón Lanfré -SW de la provincia de Río Negro- (ver Caruso, 2012, 2013) se decidió realizar un trabajo experimental y estudio cuantitativo de la madera carbonizada. El estudio antracologico de Paredón Lanfré reveló una notable diferencia entre el porcentaje de carbones de coníferas agrietados, en comparación con dos especies del género Nothofagus: - Fitzroya cupressoides (Molina) I.M. Johnst. -alerce$(39,76 \%)$,

- Austrocedrus chilensis (d. Don) pic. Serm. \& Bizzarri -ciprés de la cordillera- $(29,46 \%)$,

- Nothofagus antarctica (g. Forster) oerst. -ñire$(1,75 \%)$ y

- Nothofagus dombeyi (mirb.) Oerts. -coihue- (3,13\%).

El presente trabajo tiene como objetivo general obtener datos experimentales que permitan conocer las propiedades combustibles y entender el comportamiento que poseen ante el fuego especies nativas del bosque andino-patagónico. En este artículo 
se exponen únicamente, los datos experimentales correspondientes a las mismas especies documentadas en el sitio arqueológico Paredón Lanfré: Austrocedrus chilensis fitzroya cupressoides, Nothofagus antarctica y Nothofagus dombeyi.

Por otro lado, el trabajo experimental posee como objetivo específico: explorar si las grietas de contracción presentes en los carbones experimentales responden al estado de la madera puesta al fuego, es decir madera verde o seca. Las alteraciones producidas durante el proceso de combustión -grietas de contracción, vitrificación- dependerán del estado de la madera en el momento de su combustión (seco o verde y sano o degradado) y de las condiciones en las que se produzca el acto de la propia combustión (Zicherman, 1981; Prior \& Alvin, 1983, 1986; 1993; Schott et al., 2000; Pastor-Villegas et al., 2007). Maderas verdes o degradadas por el ataque de microorganismos no se trasformarán de la misma manera por la acción del fuego. Por esta razón, el análisis de las alteraciones permite, por un lado, estudiar las distintas modalidades de obtención del material leñoso por parte de los distintos grupos humanos y, por otro, comprender la transformación del material leñoso (Théry Parisot ,1998; Caruso \& Théry-Parisot 2011, entre otros).

El planteamiento y desarrollo de las distintas experimentaciones posibilitará recrear determinadas situaciones que enriquecerán la interpretación de los resultados obtenidos en el análisis de los sitios arqueológicos patagónicos (March 1992, Caruso 2008, 2010, 2012, Caruso \& Théry-Parisot, 2011, Caruso \& Iriarte, 2013).

A continuación se presentan el desarrollo de los distintos fuegos experimentales, y el análisis e interpretación de las grietas de contracción producidas en los carbones de las cuatro especies leñosas.

\section{MATERIAL Y MÉTODOS}

El trabajo experimental se estructura en dos partes. Una primera caracterizada por la realización de una serie de combustiones controladas con madera verde y seca de Austrocedrus chilensis, Fitzroya cupressoides, Nothofagus antarctica y Nothofagus dombeyi. Esta etapa del trabajo se basa en el estudio de las propiedades combustibles de las especies, teniendo como eje de análisis la temperatura, la duración de la combustión y de la llama, además del estado las maderas utilizadas (verde, seco). La segunda parte del trabajo se centra en el estudio de los carbones procedentes de cada una de las combustiones experimentales. La labor consistió en la cuantificación de las grietas de contracción existente en cada uno de los carbones, en la toma de medidas (largo y ancho) del plano transversal de cada fragmento y en el cálculo de la media de grietas de contracción $/ \mathrm{mm}^{2}$ para cada uno de ellos.

\section{Primera parte: combustiones controladas}

La madera utilizada en cada uno de los fuegos fue obtenida en dos períodos diferentes. En el año 2010 se realizó una primera colecta de madera, correspondiente a las cuatro especies leñosas. Un año más tarde, ya seco el material obtenido el año anterior, se procedió a la segunda colecta y desarrollo de las combustiones experimentales. Es preciso aclarar que en el caso de Fitzroya cupressoides fue utilizada madera de poda natural ${ }^{1}$. Es importante mencionar que para el estudio de las alteraciones de la madera y sus propiedades de combustión, es recomendable realizar fuegos con madera de una misma especie, procedente de distintos individuos. Sin embargo, el hecho de trabajar con una especie protegida $-F$. cupressoides- generó modificaciones en el protocolo de actuación. Razón por la cual, los fuegos de las cuatro especies leñosas fueron realizados con madera de un mismo individuo, de esta manera no sólo se intensificó el cuidado en lo que respecta a $F$. cupressoides si no que también se redujo la alteración de los resultados.

Las cuatro especies nativas utilizadas en las distintas combustiones experimentales presentan las siguientes características:

1) Fitzroya cupressoides -Fig. 1a- es una de las tres coníferas argentino-chilenas. Se caracteriza por ser el árbol de mayores dimensiones y el más longevo de Sudamérica (Lara \& Villalba 1993). Habita en la región más húmeda de los bosques andino-patagónicos, desde los $39^{\circ}$ hasta los $43^{\circ}$ 30' lat. S (Veblen \& Ashton 1982, Grosfeld, 2002).

${ }^{1}$ En el caso de Fitzroya cupressoides, para los fuegos con madera verde se utilizó madera de poda (la forma de proceder con esta especie fue consultada con personal de la Administración Parques Nacionales, Delegación Regional Patagonia. Bariloche, Argentina). 
En argentina forma bosques puros o mixtos entre 300 y 1.500 msnm (Donoso et al., 2006). Fitzroya cupressoides se asocia con Pilgerodendron uviferum, pero suele entremezclarse con Nothofagus antactica, Nothofagus dombeyi, Drimys winteri, entre otras especies. No obstante, en la parte más oriental de su distribución - por debajo de los 500 m snm- existen ejemplares relativamente aislados en las márgenes de ríos y lagunas (Donoso et al., 2006).

2) Austrocedrus chilensis -Fig. 1b-es uno de árboles característicos de los bosques del norte de la Patagonia. Es la conífera nativa de más amplia distribución latitudinal de Chile y Argentina: 39 30' S y los 43 44' S (Donoso 2006, Gallo et al., 2004). Muestra gran capacidad de asociación con otras especies arbóreas, constituyendo tipos forestales muy disímiles. La asociación con otras especies varía en función de la humedad desde las partes altas de la cordillera oriental de los Andes hacia la estepa. En las zonas más húmedas de la cordillera se asocia con Nothofagus dombeyi, formando "bosques mixtos". Con la disminución de la precipitación los rodales de austrocedrus se hacen puros convirtiéndose en "bosques compactos". Hacia la estepa las precipitaciones son menores y los bosques puros de Austrocedrus, cada vez menos densos, se transforman en "bosques marginales" (Dezzotti \& Sancholuz, 1991).
3) Nothofagus antarctica-Fig. 1d- es una especie monoica que varía de tamaño según las condiciones de hábitat. Puede poseer forma de árbol, de 10 a 15 metros de altura, o la de un pequeño subarbusto de unos cuantos decímetros (Vidal \& Premoli 2004). Entre las especies de Nothofagus sudamericano Nothofagus antarctica muestra la mayor amplitud ecológica. Su distribución geográfica se encuentra desde los $36^{\circ} 30^{\prime}$ 's hasta los $56^{\circ}$ a lo largo de la Cordillera de los Andes tanto de Chile como de Argentina (Donoso et al., 2004). En las proximidades de la estepa patagónica, donde las precipitaciones son más escasas, esta especie crece junto a austrocedrus chilensis en bosques más xéricos y abiertos. En ambientes anegadizos y turberas $N$. antarctica puede encontrarse asociado con especies como Pilgerodendrum uviferum, Drimys winteri y Fitzroya cupressoides. En Tierra del Fuego se lo encuentra junto a Nothofagus betuloides y Nothofagus pumilio (Vidal \& Premoli, 2004).

4) Nothofagus dombeyi -Fig. 1c- es un árbol monoico, que puede alcanzar entre 2,5 y 4 metros de diámetro y una altura aproximada de 40-45 metros (Díaz Vas, 1987). Su distribución en Argentina se encuentra entre los paralelos $38^{\circ} 30^{\prime} y$ $44^{\circ} \mathrm{s}$. El principal limitante para el desarrollo de esta especie es la humedad (Donoso et al., 2004). Al este de la cordillera de los Andes, en territorio argentino,

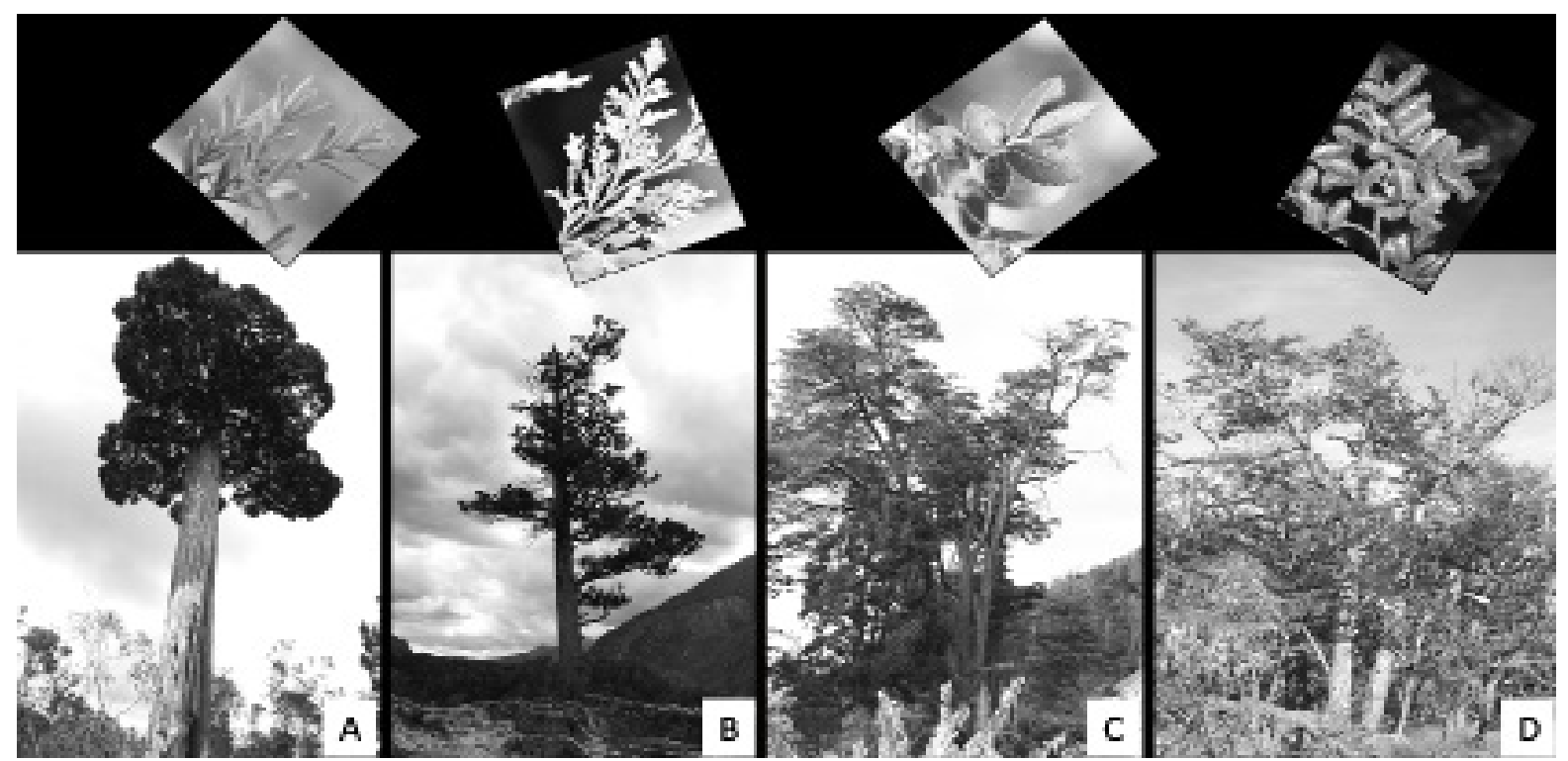

Fig. 1.A. Fitzroya cupressoides, B. Austrocedrus chilensis, C. Nothofagus dombeyi y D. Nothofagus antarctica. 
los bosques en que participa Nothofagus dombeyi son en general puros en mosaico o con algún grado de asociación con Nothofagus oblicua -roble- y Nothofagus nervosa -rauli- (Donoso, 1987). Hasta los $44^{\circ} \mathrm{S}$ donde la precipitación disminuye desde $2.500 \mathrm{~mm}$ hasta $1.800 \mathrm{~mm}$ dombeyi forma bosques mixtos junto a Austrocedrus chilensis (Dezzotti \& Sancholuz 1991, Gallo et al., 2004).

Se llevó a cabo un total de 16 combustiones, cuatro por cada una de las especies leñosas. Dos de los cuatro fuegos se efectuaron con ramas secas y dos con ramas verdes. Todas las combustiones fueron realizadas en un hogar abierto, en condiciones de laboratorio, con el objeto de limitar la incidencia de factores externos tales como la humedad atmosférica, viento, etc., que pudieran incidir en la misma. En todos los casos se siguió el mismo protocolo de acción. La madera fue medida y pesada antes y
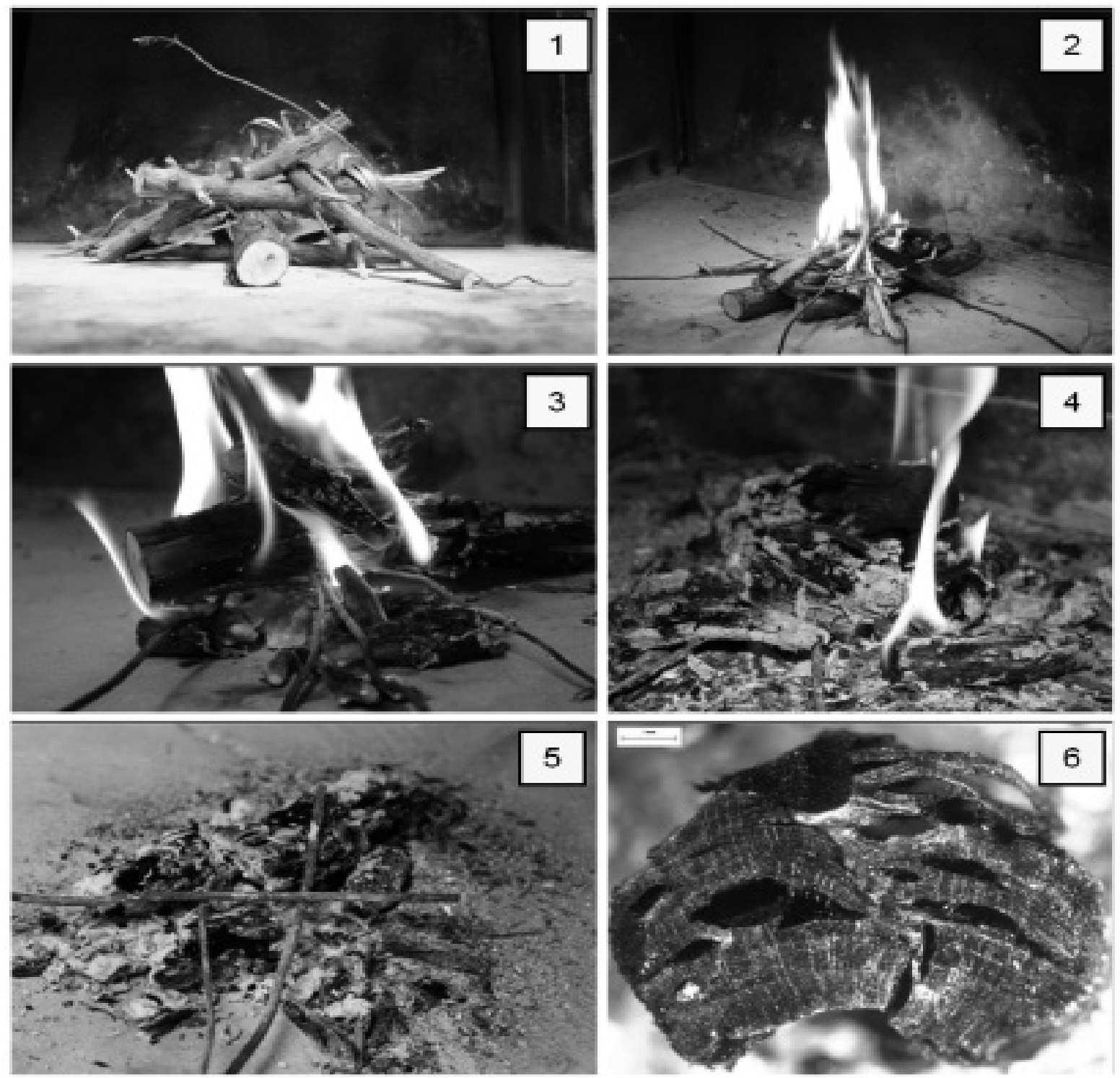

Fig. 2: Distintos momentos de una combustión experimental. 
Tabla 1. Volumen inicial y estado de maderas de las cuatro especies utilizadas en los 16 fuegos experimentales.

\begin{tabular}{|c|c|c|c|}
\hline Taxón & $\begin{array}{l}\text { Estado } \\
\text { de la } \\
\text { madera }\end{array}$ & $\begin{array}{c}\mathrm{N}^{\circ} \\
\text { muestra }\end{array}$ & $\begin{array}{c}\text { Volumen } \\
\text { inicial } \\
\left(\mathrm{cm}^{3}\right)\end{array}$ \\
\hline \multirow{4}{*}{ Fitzroya cupressoides } & \multirow{2}{*}{ Seca } & 1 & 516,04 \\
\hline & & 2 & 533,86 \\
\hline & \multirow{2}{*}{ Verde } & 1 & 461,20 \\
\hline & & 2 & 467,84 \\
\hline \multirow{4}{*}{ Austrocedrus chilensis } & \multirow{2}{*}{ Seca } & 1 & 720,17 \\
\hline & & 2 & 536,86 \\
\hline & \multirow{2}{*}{ Verde } & 1 & 552,71 \\
\hline & & 2 & 386,63 \\
\hline \multirow{4}{*}{ Nothofagus dombeyi } & \multirow{2}{*}{ Seca } & 1 & 939,51 \\
\hline & & 2 & 900,43 \\
\hline & \multirow{2}{*}{ Verde } & 1 & 1259,41 \\
\hline & & 2 & 1181,87 \\
\hline \multirow{4}{*}{ Nothofagus antarctica } & \multirow{2}{*}{ Seca } & 1 & 1429,8 \\
\hline & & 2 & 1284,71 \\
\hline & \multirow{2}{*}{ Verde } & 1 & 1191,11 \\
\hline & & 2 & 1037,83 \\
\hline
\end{tabular}

después de la combustión (Tabla 1); la temperatura, duración de la flama y duración total de cada uno de los fuegos, fueron registradas durante todo el proceso de combustión (Caruso \& Théry-Parisot, 2011) -ver Figura 2 (1-2-3-4-5-6)

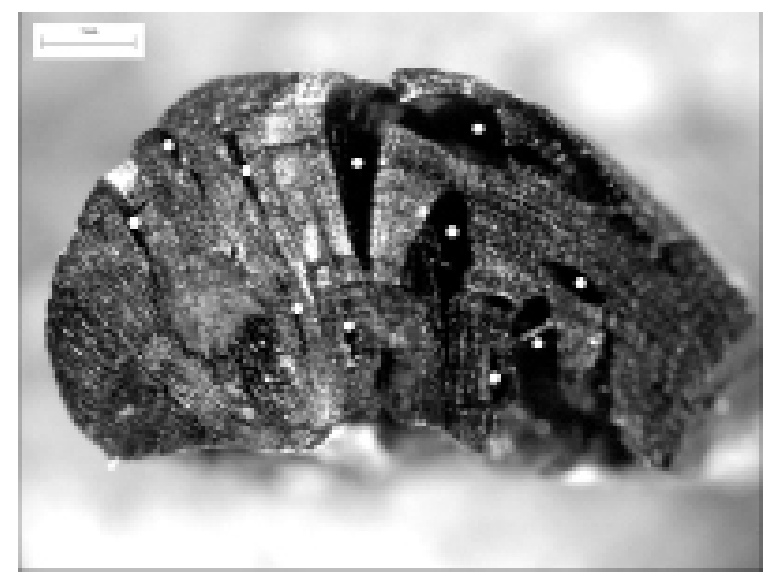

Fig. 3. Cuantificación de grietas sobre el plano transversal del carbón.
Segunda parte: estudio de los carbones experimentales

La segunda parte del trabajo consistió en el estudio de los carbones procedentes de las distintas combustiones experimentales. Se estudió un total de 400 fragmentos, 25 por cada uno de los fuegos.

Las grietas de contracción fueron estudiadas a través de un analizador de imágenes image pro-plus. El plano transversal de cada carbón fue fotografiado utilizando una cámara conectada a un binocular leica (mz6). Sobre cada una de las imágenes realizadas se procedió a la cuantificación de las grietas de contracción presentes en cada uno de los fragmentos (ver Fig. 3). En cada carbón también se midió el largo $y$ ancho del plano transversal, medidas necesarias para poder calcular la superficie de cada fragmento.

El porcentaje de grietas de contracción no tiene valor diagnóstico en el seno de una muestra, sin embargo el cálculo del número medio de grietas de contracción $/ \mathrm{mm}^{2}$ es un método que permite discriminar la combustión de madera verde o madera seca, tanto en material experimental como arqueológico (Caruso 2012, 2013, Caruso \& Thèry -Parisot 2011, Thèry-Parisot \& Henry, 2012). De esta manera, sobre la base del número de grietas de contracción y la superficie de cada fragmento fue posible calcular el número medio de grietas de contracción $/ \mathrm{mm}^{2}$ para cada uno de los carbones -Tabla 2-.

\section{RESULTADOS}

\section{Primera parte}

El tiempo total de combustión alcanzado por las 16 combustiones experimentales fue de 1.14hs a 3.04hs. Entre los fuegos de Nothofagus antarctica y Fitzroya Cupressoides la mayor duración de combustión se registró en aquellos realizados con madera seca, en comparación a los efectuados con madera verde. Por el contrario, en el caso de Austrocedrus chilensis, las combustiones efectuadas con madera verde duraron más tiempo que las de madera seca. Por lo que respecta a Nothofagus dombeyi los dos fuegos que registraron una mayor duración de combustión se llevaron a cabo con madera verde y con madera seca (Tabla 3 ).

En cuanto a la duración total de la llama, los 
Tabla 2. Ejemplo del cálculo del número medio de grietas de contracción $/ \mathrm{mm}^{2}$ para algunos carbones experimentales de Nothofagus antarctica

\begin{tabular}{|c|c|c|c|c|c|}
\hline $\begin{array}{c}\mathrm{N}^{\circ} \\
\text { carbón }\end{array}$ & $\begin{array}{c}\text { Longitud } \\
(\mathrm{mm})\end{array}$ & $\begin{array}{c}\text { Ancho } \\
(\mathrm{mm})\end{array}$ & superficie & $\begin{array}{c}\mathrm{N}^{\circ} \\
\text { grietas }\end{array}$ & $\begin{array}{c}\mathrm{N}^{\circ} \text { medio de } \\
\text { grietas/100 } \mathrm{mm}^{2}\end{array}$ \\
\hline 1 & 10,83 & 12,65 & 137 & 17 & 12,41 \\
\hline 2 & 9,09 & 16,61 & 150,98 & 16 & 10,6 \\
\hline 3 & 6,14 & 13,01 & 79,88 & 74 & 92,64 \\
\hline 4 & 6,7 & 10,88 & 72,9 & 9 & 12,35 \\
\hline 5 & 7,78 & 13,02 & 101,3 & 1 & 0,99 \\
\hline
\end{tabular}

fuegos realizados con madera seca de Austrocedrus chilensis y Fitzoroya cupressoides evidenciaron una llama más duradera, que los de madera verde. En el caso de Nothofagus dombeyi, por el contrario, la llama con mayor duración se observó en las combustiones de madera verde. Finalmente entre los fuegos de Nothofagus antarctica el mayor tiempo de duración de la llama fue evidente tanto en combustiones de madera seca como de madera verde.

Como se observa en la tabla que se presenta a continuación (Tabla 3), los resultados de las distintas combustiones permiten apreciar que la duración total de la combustión así como la de las llamas no parece tener relación con el estado de la madera (verde-seco) de las diferentes especies nativas utilizadas en los 16 fuegos experimentales.

La temperatura máxima alcanzada por los distintos fuegos fue de $786^{\circ}$ y de $426^{\circ}$ la mínima.

Tabla 3. Resultados de las distintas combustiones experimentales (ordenados por temperatura máxima alcanzada)

\begin{tabular}{|c|c|c|c|c|c|c|}
\hline Taxón & $\begin{array}{l}\text { Estado } \\
\text { de la } \\
\text { madera }\end{array}$ & $\begin{array}{c}\mathrm{N}^{\circ} \\
\text { muestra }\end{array}$ & $\begin{array}{l}\text { Duración } \\
\text { de la } \\
\text { combustión } \\
\text { (en min) }\end{array}$ & $\begin{array}{c}\text { Duración } \\
\text { de la } \\
\text { llama } \\
\text { (en min) }\end{array}$ & $\begin{array}{c}\mathrm{T}^{\circ} \\
\text { máxima }\end{array}$ & $\begin{array}{c}\text { Residuos } \\
\text { (en g) }\end{array}$ \\
\hline \multirow{4}{*}{ Austrocedrus chilensis } & \multirow{2}{*}{ Seca } & 1 & 74 & 25 & $497^{\circ}$ & 18,67 \\
\hline & & 2 & 80 & 28 & $488^{\circ}$ & 15,26 \\
\hline & \multirow{2}{*}{ Verde } & 1 & 86 & 13 & $464^{\circ}$ & 19,68 \\
\hline & & 2 & 118 & 12 & $426^{\circ}$ & 18,14 \\
\hline \multirow{4}{*}{ Fitzroya cupressoides } & Seca & 1 & 107 & 45 & $583^{\circ}$ & 37,56 \\
\hline & Verde & 2 & 90 & 12 & $522^{\circ}$ & 15,86 \\
\hline & Seca & 2 & 98 & 31 & $459^{\circ}$ & 18,21 \\
\hline & Verde & 1 & 80 & 13 & $441^{\circ}$ & 55,7 \\
\hline \multirow{4}{*}{ Nothofagus dombeyi } & \multirow{2}{*}{ Seca } & 2 & 134 & 37 & $735^{\circ}$ & 23,69 \\
\hline & & 1 & 138 & 22 & $696^{\circ}$ & 26,88 \\
\hline & \multirow{2}{*}{ Verde } & 1 & 140 & 40 & $693^{\circ}$ & 41,83 \\
\hline & & 2 & 137 & 40 & $647^{\circ}$ & 41,56 \\
\hline \multirow{4}{*}{ Nothofagus antarctica } & Seca & 1 & 184 & 50 & $786^{\circ}$ & 111,34 \\
\hline & \multirow{2}{*}{ Verde } & 1 & 156 & 50 & $783^{\circ}$ & 68,99 \\
\hline & & 2 & 140 & 45 & $773^{\circ}$ & 63,36 \\
\hline & Seca & 2 & 170 & 58 & $768^{\circ}$ & 77,4 \\
\hline
\end{tabular}


Las combustiones llevadas a cabo con madera de Nothofagus registraron temperaturas más altas que aquellas realizadas con madera de coníferas (F. cupressoides y A. chilensis). Los fuegos de Nothofagus antarctica y nothofagus dombeyi evidenciaron temperaturas próximas a los $750^{\circ}-800^{\circ}$ (fig. 4), mientras que los de Fitzroya cupressoides y Austrocedrus chilensis a los $500^{\circ}-600^{\circ}$-ver Tabla 3-. En cuanto al estado de la madera, las combustiones efectuadas con madera seca de Austrocedrus chilensis y Nothofagus dombeyi alcanzaron temperaturas superiores en comparación a aquellas realizados con madera verde de estas mismas especies. Por el contrario, en el caso de Nothofagus antarctica y Fitzoroya cupressoides las temperaturas máximas se registraron tanto en fuegos efectuados con madera verde como con madera seca.

Segunda parte

Todos los carbones producto de las 16 combustiones experimentales presentan grietas de contracción. No obstante, en el caso de Austrocedrus chilensis existe una marcada diferencia entre la media de grietas de contracción de carbones producto de combustiones con madera verde $\left(17,35 / 100 \mathrm{~mm}^{2} 17,3 / 100 \mathrm{~mm}^{2}\right)$ y la correspondiente a los carbones procedentes de fuegos efectuados con madera seca $\left(6,21 / 100 \mathrm{~mm}^{2}\right.$ y $6,19 / 100 \mathrm{~mm}^{2}$ ) -tabla 4-. La misma situación se evidencia en las muestras de Fitzroya cupressoides, donde los carbones resultado de combustiones con madera verde poseen una media de grieta de: $15,33 / 100 \mathrm{~mm}^{2}$ y $12,01 / 100 \mathrm{~mm}^{2}$, mientras que aquellos procedentes de combustiones con madera seca tienen una media más baja: 7,54/100mm² y $7,06 / 100 \mathrm{~mm}^{2}$.

Los resultados de los fuegos realizados con madera de Nothofagus antarctica y Nothofagus dombeyi ofrecen un panorama totalmente diferente al de las coníferas. En el caso de los Nothofagus no se evidencian marcadas diferencias entre los fuegos realizados con madera seca y verde. La media de grietas de contracción para los carbones de Nothofagus antarctica, producto de los fuegos

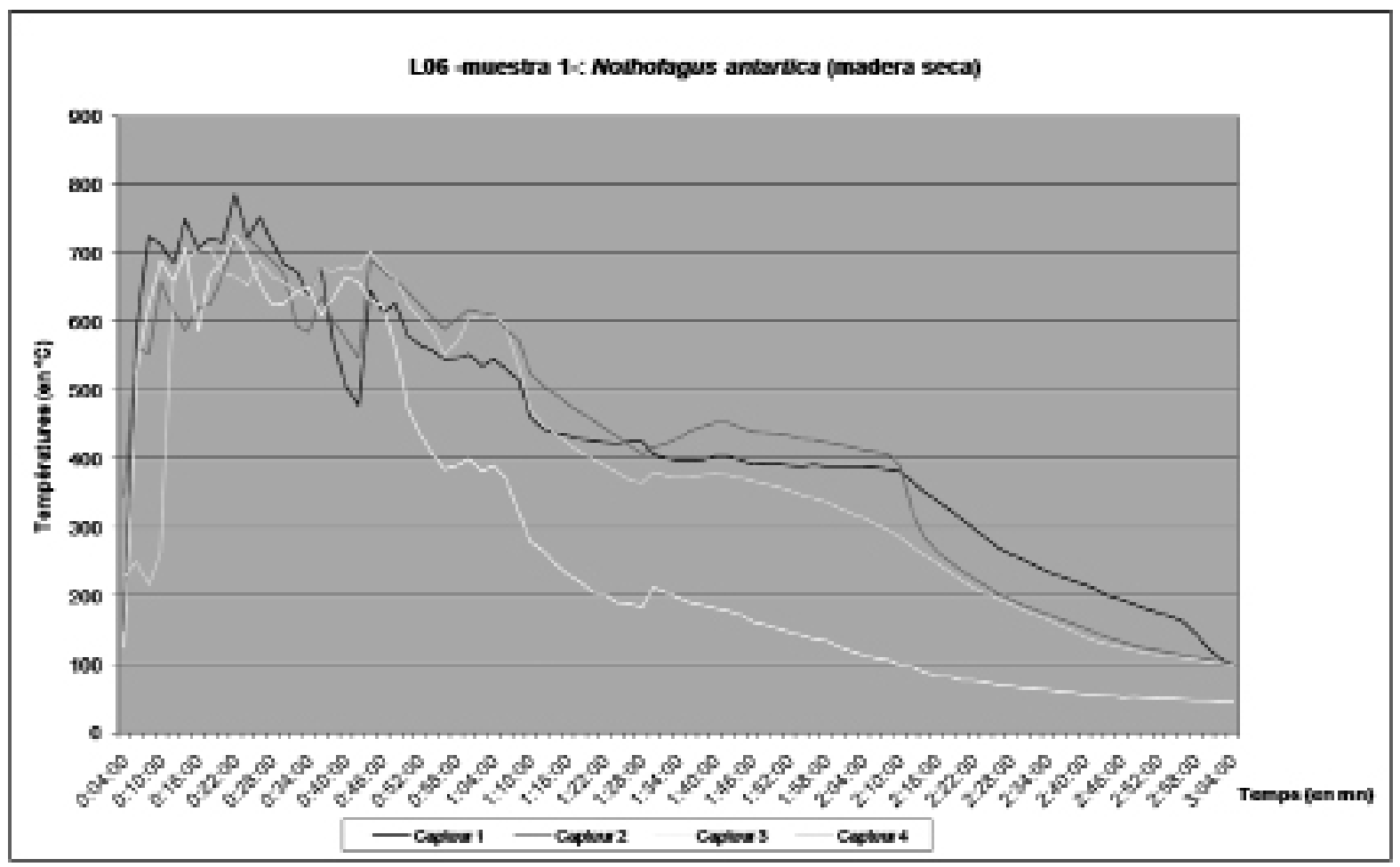

Fig. 4. Registro de la temperatura, cada 6 minutos, de toda la combustión experimental de madera seca de Nothofagus antarctica. 
Tabla 4.Registro de media de grietas de contracción $/ \mathrm{mm}^{2}$ por especie vegetal, según el estado fisiológico de la madera utilizada en cada fuego experimental.

\begin{tabular}{|c|c|c|c|}
\hline Taxón & $\begin{array}{c}\text { Estado } \\
\text { de la } \\
\text { madera }\end{array}$ & $\begin{array}{c}\mathrm{N}^{\circ} \\
\text { muestra }\end{array}$ & $\begin{array}{c}\text { Media de } \\
\text { grietas } / 100 \mathrm{~mm}^{2}\end{array}$ \\
\hline \multirow{4}{*}{$\begin{array}{l}\text { Austrocedrus } \\
\text { chilensis }\end{array}$} & \multirow{2}{*}{ Verde } & 1 & 17,35 \\
\hline & & 2 & 17,3 \\
\hline & \multirow{2}{*}{ Seco } & 1 & 9,19 \\
\hline & & 2 & 6,21 \\
\hline \multirow{4}{*}{$\begin{array}{c}\text { Fitzroya } \\
\text { cupressoides }\end{array}$} & \multirow{2}{*}{ Verde } & 1 & 15,33 \\
\hline & & 2 & 12,01 \\
\hline & \multirow{2}{*}{ Seco } & 1 & 7,54 \\
\hline & & 2 & 7,06 \\
\hline \multirow{4}{*}{$\begin{array}{l}\text { Nothofagus } \\
\text { antarctica }\end{array}$} & \multirow{2}{*}{ Verde } & 1 & 12,28 \\
\hline & & 2 & 18,43 \\
\hline & \multirow{2}{*}{ Seco } & 1 & 29,39 \\
\hline & & 2 & 19,41 \\
\hline \multirow{4}{*}{$\begin{array}{c}\text { Nothofagus } \\
\text { dombeyi }\end{array}$} & \multirow{3}{*}{ Verde } & 1 & 10,89 \\
\hline & & 2 & 5,67 \\
\hline & & 1 & 9,5 \\
\hline & Seco & 2 & 10,4 \\
\hline
\end{tabular}

realizados con madera seca es de $18,43 / 100 \mathrm{~mm}^{2}$, mientras que la media para los carbones de las combustiones con madera verde es de 19,41/100 $\mathrm{mm}^{2}$.

Un cuadro similar se aprecia en el estudio de los carbones de Nothofagus dombeyi donde la media de grietas de contracción para los carbones de fuegos con madera verde es de $10,89 / 100 \mathrm{~mm}^{2}$, y $10,40 / 100 \mathrm{~mm}^{2}$ para los de madera seca (ver Tabla 4). La aplicación de la prueba exacta de fisher no evidencia diferencias estadísticamente significativas entre los carbones producto de combustiones realizadas con madera seca y madera verde de Nothofagus antarctica y Nothofagus dombeyi (Fig. 5-6).

\section{DISCUSIÓN Y CONCLUSIÓN}

Toda experimentación sobre el pasado, tanto en sus dimensiones socioculturales -tecnología, funcionalidad, etc.- como en lo relativo a la conservación de las evidencias de ese pasado, se desarrollará enmarcada por parámetros que simplifican directa o
Test PLSD de Fisher para $\mathrm{n}^{\circ}$ grietas de contracción $/ \mathrm{mm}^{2}$ Effet : caté

Nive1 de significatividad : $5 \%$

Exclusion de lignes: Données sans titre $\# 1$.sud

\begin{tabular}{|c|c|c|c|}
\hline & Diff. moy. & Diff. crit. & Valor $p$ \\
\hline $\mathrm{ABS}, \mathrm{ABV}$ & $-9,621$ & 5,596 &, 0008 \\
\hline ABS, FBS & 401 & 5,596 & 8880 \\
\hline ABS, FBV & $-6,039$ & 5,654 & .0364 \\
\hline ABS, NABS & -16.699 & 5.596 & $<, 0001$ \\
\hline ABS, NABV & -7.652 & 5,596 & .0075 \\
\hline ABS, NDS & $-2,245$ & 5.596 & .4307 \\
\hline ABS, NDV & -.575 & 5.596 & 8399 \\
\hline $\mathrm{ABV}, \mathrm{FBS}$ & 10,022 & 5,596 &, 0005 \\
\hline$A B Y, F B V$ & 3,582 & 5,654 & 2137 \\
\hline ABV. NABS & -7.078 & 5.596 & .0133 \\
\hline ABV, NABV & 1,969 & 5.596 & .4894 \\
\hline ABY, NDS & 7,376 & 5,596 &, 0099 \\
\hline A BV, NDV & 9.046 & 5,596 & .0016 \\
\hline FBS, FBV & $-6,440$ & 5,654 &, 0257 \\
\hline FBS, NABS & $-17,100$ & 5,596 & $<, 0001$ \\
\hline FBS, NABV & $-8,053$ & 5,596 & .0049 \\
\hline FBS, NDS & $-2,647$ & 5.596 & .3530 \\
\hline FBS, NDV & -977 & 5,596 & .7317 \\
\hline FBV, NABS & $-10,659$ & 5,654 & 0002 \\
\hline FBV, NABV & $-1,612$ & 5,654 &, 5754 \\
\hline FBV, NDS & 3,794 & 5,654 & .1879 \\
\hline FBV, NDV & 5,464 & 5,654 & .0582 \\
\hline NABS, NABV & 9,047 & 5.596 & .0016 \\
\hline NABS, NDS & 14,453 & 5,596 & $<, 0001$ \\
\hline NABS, NDV & 16,123 & 5,596 & $<, 0001$ \\
\hline NABV, NDS & 406 & 5,596 & 0583 \\
\hline NABV, NDY & 6 & 596 & 0133 \\
\hline NDS, NDV & 1,670 & 5,596 & 5577 \\
\hline
\end{tabular}

Fig. 5. Cálculo de media de grietas de contracción por cada una de las especies nativas.

pueden ser resueltas mediante enfoques experimentales adaptados a cada caso de estudio (Pellegrin, 2011). La realización de combustiones experimentales, y el estudio de los carbones procedentes de cada uno de los fuegos, permitieron profundizar en el conocimiento de las características de cuatro especies nativas de los bosques patagónicos: Austrocedrus chilensis, Fitzroya cupressoides, Nothofagus antarctica y Nothofagus dombeyi.

El desarrollo y estudio de las distintas combustiones experimentales permitió apreciar que la duración de las mismas así como la de las llamas, no mantiene relación con el estado de la madera (verde-seco) utilizada como combustible. Por otro lado, la temperatura más alta alcanzada en cada uno de los fuegos, tampoco demuestra 
Tableau ANOVA para grietas de contracción $/ \mathrm{m} \mathrm{m}^{2}$ Exclusion de lignes : Données sans titre \#1.svd

\begin{tabular}{l|r|r|r|r|r|r|r|}
\multicolumn{1}{l}{} & \multicolumn{2}{c}{ ddl } & \multicolumn{2}{c}{ Somme des carrés } & \multicolumn{2}{c}{ Carré moyen } & \multicolumn{2}{c}{ Valeur de $F$} & \multicolumn{2}{c}{ Valeur de $p$} & \multicolumn{2}{c}{ Lambda } & \multicolumn{2}{c}{ Puissance } \\
\cline { 2 - 8 } caté & 7 & 12345,188 & 1763,598 & 8,707 & $<, 0001$ & 60,952 & 1,000 \\
\cline { 2 - 8 } Résidu & 390 & 78990,158 & 202,539 & & & & \\
\cline { 2 - 8 }
\end{tabular}

Cuadro de media de grietas de contracción $/ \mathrm{m} \mathrm{m}^{2}$ Effet : caté

Exclusion de lignes: Données sans titre \#1.svd

\begin{tabular}{l|r|r|r|r|}
\multicolumn{1}{c}{} & \multicolumn{1}{c}{ Número } & \multicolumn{1}{c}{ Media } & Desv. estand & Err. estand. \\
\cline { 2 - 5 } Ac (ms) & 50 & 7,705 & 6,465 &, 914 \\
\cline { 2 - 5 } Ac (mv) & 50 & 17,326 & 15,907 & 2,250 \\
\cline { 2 - 5 } Fc (ms) & 50 & 7,304 & 5,488 &, 776 \\
\cline { 2 - 5 } Fc (mv) & 48 & 13,745 & 6,523 &, 941 \\
\cline { 2 - 5 } $\mathrm{Na}(\mathrm{ms})$ & 50 & 24,404 & 24,973 & 3,532 \\
\cline { 2 - 5 } $\mathrm{Na}(\mathrm{mv})$ & 50 & 15,357 & 17,020 & 2,407 \\
$\mathrm{Nd}(\mathrm{ms})$ & 50 & 9,951 & 13,458 & 1,903 \\
\cline { 2 - 5 } $\mathrm{Nd}(\mathrm{mv})$ & 50 & 8,281 & 12,322 & 1,743 \\
\cline { 2 - 5 } & \multicolumn{4}{|c}{}
\end{tabular}

Ac (ms): Austrocedrus chilensis (madera seca) Ac (mv): Austrocedrus chilensis (madera verde) Fc (ms): Fitzroya cupressoides (madera seca) Fc (mv): Fitzroya cupressoides (madera verde) $\mathrm{Na}(\mathrm{ms})$ : Nothofagus antarctica (madera seca) $\mathrm{Na}(\mathrm{mv})$ : Nothofagus antarctica (madera verde) $\mathrm{Nd}$ (ms): Nothofagus dombeyi (madera seca) $\mathrm{Nd}(\mathrm{mv})$ : Nothofagus dombeyi (madera verde)
Gráfico des interactions pour $\mathbf{n}$ fente $/ \mathrm{m} \mathrm{m}^{2}$ Effet : caté

Exclusion de lignes: Données sans titre \#1.svd

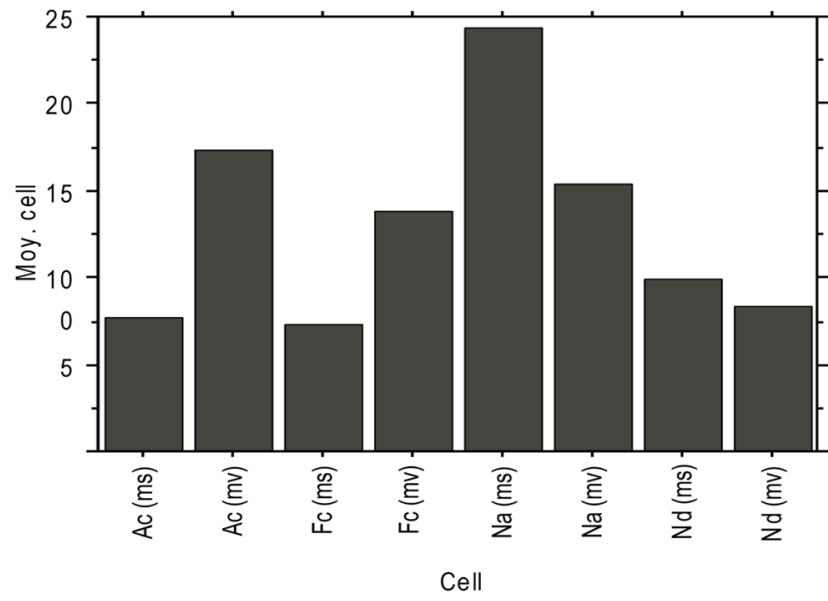

Fig. 6. Test de Fisher para el número de grietas de contracción $/ \mathrm{mm}^{2}$ en los carbones de las cuatro especies nativas.

una relación directa con la presencia de grietas de contracción registradas en los carbones de las 16 combustiones realizadas.

Los fuegos experimentales y el estudio de las grietas de contracción presentes en los carbones, demostraron diferencias entre las especies leñosas estudiadas. Las distintas combustiones experimentales aquí descriptas permiten apreciar, en algunos casos, que la carbonización de madera saturada en agua favorece el aumento substancial del numero de grietas de contracción. Este fenómeno fue claramente observado entre las combustiones realizadas con madera verde de Austrocedrus chilensis y Fitzroya cupresoides. Los resultados señalan, que el número de grietas $/ \mathrm{mm}^{2}$ es mayor entre los carbones producto de madera verde que entre los obtenidos con madera seca. Por lo tanto, en Austrocedrus chilensis y Fitzroya cupresoides el número de grietas de contracción depende del contenido de humedad de la madera utilizada como combustible. Por el contrario, en el caso de Nothofagus antarctica y
Nothofagus dombeyi los resultados alcanzados no evidencian diferencias estadísticamente significativas entre los fuegos realizados con madera verde $y$ seca. En consecuencia, la aparición de grietas de contracción en los carbones de estas especies, no depende de la humedad inicial de la madera, por lo que no debería ser considerado como un signo diagnóstico de esta variable antes de que se lleve a cabo la combustión. En síntesis, el número de grietas contracción $/ \mathrm{mm}^{2}$ permite discriminar la utilización de madera seca y de madera verde entre las gimnospermas -Austrocedus chilensis y Fitzroya cupressoides-, mientras que esta diferencia no es tan clara en las angiospermas -nothofagus antarctica y nothofagus dombeyi- (Caruso 2012, 2013, Caruso \& Théry-Parisot, 2011). Esta diferencia se explicaría a partir de la mayor variabilidad y complejidad anatómica de las angiospermas, constituidas por células, vasos conductores de savia, tejido de sostén, fibras y parénquima, en comparación con árboles más primitivos como lo son las gimnospermas. Estas 
últimas con un sistema axial caracterizado por una estructura homóxila y ausencia de vasos (Chamberlain 1934, Eames 1960, Fahn 1982).

El estudio detallado del fenómeno de las grietas de contracción demuestra un comportamiento diferencial relacionado con las especies experimentadas. Este hecho puede deberse a distintas razones; en primer lugar, a que cada especie vegetal posee componentes anatómicos que les son propios y que son responsables de su comportamiento ante el fuego. En segundo lugar, puede existir una gran variabilidad anatómica para una misma especie, que conlleva un comportamiento diferencial durante la carbonización de la madera. Finalmente las características anatómicas de una misma especie pueden variar en función de la posición en la que se encuentren dentro del mismo árbol y de la misma orientación de la muestra -en relación con la disposición de los principales elementos anatómicos de la madera- (Théry-Parisot, 2001). Según Prior \& Alvin $(1986,1993)$ las mismas características anatómicas de la madera serán las que determinen su comportamiento durante la combustión y la interacción con factores tales como temperatura, duración de la carbonización, y tasa inicial de humedad, entre otros.

El trabajo experimental, no solo permite profundizar en las características que poseen las distintas especies leñosas como combustible y su comportamiento ante el calor del fuego, sino que posibilitara recrear determinadas situaciones que ayudan a comprender y discutir los resultados arqueobotánicos. En el caso concreto de los resultados antracologicos de Pardón Lanfré, los resultados experimentales obtenidos, permitieron discutir sobre las modalidades de adquisición y uso del material leñoso por parte de los cazadores-recolectores que ocuparon este alero. Es preciso remarcar por ello, la necesidad de continuar con el desarrollo de trabajos experimentales claros y precisos orientados al estudio de: las distintas fases del proceso de combustión, alteraciones del material leñoso, procesos relacionados con el uso de la madera como materia prima para la fabricación de artefactos u objetos, etc.

En síntesis, el correcto diseño de diversos trabajos experimentales permitirá por lo tanto enriquecer el estudio sobre el uso del fuego, las distintas modalidades de adquisición y usos del material leñoso por parte de los grupos del pasado, complementando así el análisis arqueobotánico de los distintos sitios arqueológicos.

\section{AGRADECIMIENTOS}

Al Ministerio de Ciencia e Innovación de España por la beca pre-doctoral (beca FPI MICINN). A Nahuel Caruso por la búsqueda y secado de la madera utilizada en las experimentaciones. A Mirian Peloso, a la familia Sierra y a Haydée Palleres por su ayuda en la realización de los fuegos. Al Laboratoire d'Archéobotanique del CEPAM-CNRS (Laboratoire Cultures et Environnements. Préhistoire, antiquité, moyen âge), por la utilización del equipamiento necesario para el desarrollo de alguno de los estudios. A Soledad Caracotche por su continuo asesoramiento. A Isabelle Thèry-Parisot y Alain Carré (CEPAM-CNRS) por su colaboración en parte de este trabajo.

\section{BIBLIOGRAFÍA}

Allué, E., Nadal J., Estrada A. \& García-Argüelles P. (2007). Los datos antracológicos de la Balma del Gai (Bages, Barcelona): una aportación al conocimientonde la vegetación y la explotación de los recursos forestales durante el Tardiglaciar en el NE Peninsular. Trabajos de Prehistoria 64(1), 87-97.

Andreoni D., Gil A. \&CapparelliA. (2011).Efectos de la carbonización en especies leñosas de las provincias Fitogeográficas Patagónica y del Monte (Mendoza, Argentina): una perspectiva arqueológica.En: Pochettino, M.; Ladio, A. \& Arenas, P. (Eds.), Traditions and transformations in Ethnobotany (p. 33-37). San Salvador de Jujuy: CYTED, Programa Iberoamericano Ciencia y Tecnología para el Desarrollo.

Baena, J. \&Cuartero, F. (2009).Perspectivesmetodològiques de l'experimentació en Arqueología. Cota Zero 24, 21-30

Bazile-Robert, R.(1982).Données experimentales pourl'anthracoanalyse. Etudes Quaternaires Languedociennes 2,19-28.

BraadbaaF., PooleI.\&Van BrussealA.(2009)Preservation potencial of diarcoal in alMine environments: an experimental approach and implicationsforthearchaeological record. JournaI of Archaeological Science 36(8), 1672-1679.

Buxó, R. (1997).Arqueología de las plantas. Barcelona: Editorial Crítica.

Buxó, R. \& Piqué R. (2003).La recogida de muestras en arqueobotánica: objetivos y propuestas metofológicas. La gestión de los recursos vegetales y la transformación del paleopaisaje en el Mediterráneo occidental. 
Barcelon:Museud'Arqueologia deCatalunya.

Caruso, L. (2008).Los usos de la madera entre los cazadoresrecolectores Selknam de Tierra del Fuego. Barcelona: Treball de recerca-Doctoratd'ArquelogíaPrehistòrica, $U$ niversitat Autónoma de Barcelona.

Caruso, L. (2010).Ethnographie, archéobotanique et expérimentation sur le sited'Ewan I (Tierra del Fuego, Argentine). Anthropobotanica1, 3-17.

Caruso, L. (2012).Modalidades de adquisición y usos del material leñoso entre grupos cazadores-recolectores patagónicos (Argentina). Métodos y técnicas de estudio del material leñoso arqueológico. Tesis doctoral, UniversitatAutònoma de Barcelona (https://www.educacion. es/teseo/mostrarRef.do?ref=986004).

Caruso, L. (2013). Espacios interdisciplinarios en la Arqueobotánica: alcances y aportes para la investigación arqueológica en Patagonia. En:A. F. Zangrando, R. Barberena, A. Gil, G. Neme, L. Giardina, L. Luna, C. Otaola, L. Paulides, L. Salgán\& A. M. Tivoli (Eds.), Tendencias Teórico-Metodológicas y Casos de Estudio en la Arqueología de la Patagonia. Buenos Aires: Museo de Historia Natural de San Rafael, Mendoza; Sociedad de Antropología Argentina e Instituto Nacional de Antropología y Pensamiento Latinoamericano.

Caruso, L., Mansur E. \&Piqué R. (2008) Voces en el bosque: el uso de recursos vegetales entre cazadores-recolectores de la zona central de tierra del fuego. Darviniana $\mathrm{Pu}$ blicación Botánica 46(2), 202-212.

Caruso, L. \& Théry-Parisot I. (2011).Experimentation and combustion properties of PatagonianAndeanforest (Argentina). Sagutum. Extra 11, 39-40

Caruso, L. \& E Iriarte, E.(2013). Análisis de la composición química de partículas y precipitados minerales en material leñoso: madera flotada o no flotada en el sitio Orejas de Burro 1 (Santa Cruz, Patagonia Argentina). En: A. Palomo, R. Piqué \& X. Terrades (Eds.),Experimentación en arqueología. Estudio y difusión del pasado. Girona:Serie Monográfica del MAC.

Chabal, L. (1992).Représentativitépaléo-écologique des charbons de boisarchéologiquesissus du bois de feu. Dans Vernet J-L. (éd) Les charbons de bois, les anciensécosystèmes et le rôle de L'homme. Bulletin de la Société Botanique de France 139(2-4), 213-236

Chabal, L.; Fabre, J.F \&Thery- Parisot, I. (1999).Lanthracologie. En: Ferdière, A. (Ed.),La Botanique.Paris: Errance,Collections Archéologiques.

Chamberlain, C.J. (1957[1934]). Gymnospermsstructure and evolution, New York:Johnson, Reprint Corporation.

Collina Girard, J. (1993).Feu par percussion, feu par friction.
Bulletin de la Société Préhistoire Française 90 (2), 159-173.

Dezzotti, A \&Sancholuz, L.(1991). Los bosques de Austrocedruschilensis en Argentina: ubicación, estructura y crecimiento. Bosque 12(2), 43-52

Diaz-Vaz, J. E. (1987). Anatomía de la madera de Nothofagusdombeyi. Bosque 8(1), 63-65.

Donoso, C. (1987). Variación natural en especies de Nothofagus en Chile. Bosque 8 (2), 85-97.

Donoso, C., Lara, A., Escobar, B., Premoli, A. \&Souto, C.(2006). Fitzroyacupressoides (Molina) I.M. Johnst. (Alerce, Lahén, Lahuán). En: Donoso, C. (Ed.), Las especies arbóreas de los bosques templados de chile y argentina. Autoecología.Santiago: Marisa Cúneo Ediciones.

Donoso, C.; Steinke, S. \&Premoli, A. (2006).Nothofagusantarctica (G. Forster) Oerst. Nirre, Nire, Niré, Anís (Tierra del Fuego) Nirre: de Ngéré (mapudungun).En: Donoso, C. (Ed.), Las especies arbóreas de los bosques templados de chile y argentina. Autoecología.Santiago : Marisa Cúneo Ediciones.

Dufraisse, A.; Sordoillet, D. \&WellerO. (2010).Laltération des charbons de boisnéolithiques de la source de PoianaSlatineià Lunca (Neamt, Roumanie): évolutionnaturelleouconséquence des techniquesd'exploitation? En: Actes de la TableRonde, Taphonomie de la combustión des résidus organiques et des structures de combustión en contexte archéologique, 27-29 mayo 2008. Valbonne: CEPAM.

Eames, A (1960).Morphology of theangiosperms. New York: Mc Graw Hill Book Covers.

Fahn, A. (1982).PlantAnatomy. Oxford: PergamonPress.

Gallo, L. Pastorino, M. \& Donoso, C. (2004). Variación en Austrocedruschilensis (D. Don) Pic. Ser et Bizzarri (Ciprés de la Cordillera). En: Donoso, C., Premoli, A.; L. Gallo \& I. Ipinza (Eds.),Variación Intraespecífica en las especies arbóreas de los bosques templados de Chile y Argentina. Santiago: Editorial Universitaria.

Grosfeld, J. (2002).Análisis de la variabilidad morfológica y arquitectural de Austrocedruschilensis (D. Don) pic. Serm. Et Bizzarri, Fitzroyacupressoides (Molina) I.M. Johnst, Pilgerondronuviferum (d. Don) Florin y Cupressussempervivens L. (Cupressaceae). Tesis Doctoral, Universidad Nacional de Comahue, Argentina.

Hastorf, C. A. (1999).Recent Research in Paleoethnobotany. Journal of Archaeological Research 7(1), 55-103.

Lara, A. \& Villalba, R. (1993). A 3620-year temperature record fromFitzroyacupressoidestreerings in southern South America. Science 260, 1104-1106

March, R. (1992).Lutilisation du boisdans les foyers prèhistori- 
ques: une approcheexpérimentale. Bulletin de la Société Botanique de France 139, 245-255

March, R. (1996).L'Etude des structures de combustionpréhistoriques: une approcheinterdisciplinaire. XIII International Congress of Prehistoricand protohistoric Sciences ForliItalia-8/14 September 1996 Colloquia 5 TheLower and MiddlePaleolithicColloquium IX pp. 251-75. Bar Yosef, O.; Cavalli-Sforza, L.; March, R.J. et PipernoEditors.

March, R. Baldessari, A. \& Gross G. (1989). Determinación de compuestos orgánicos en estructuras de combustión arqueológicas. Nature et Funtion des foyes préhistoriques. Actes du Colloque International de Nemours. Mémoires du Musée du PréhistoireD’lle de France, 2, 47-58.

Marconetto, B. (2002). Análisis de los vestigios de combustión de los sitios Alero Don Santiago y Campo Moncada. En:C. Pérez de Micou (Ed.),Plantas y Cazadores en Patagonia.Buenos Aires: Facultad de Filosofía y Letras Universidad deBuenos Aires.

McParland L., C. Margaret, E. Collinson, M., Scott, A.C., Campbell, G. \&Veal, R. (2010).Isvitrification in charcoal a result of hightemperatureburning of wood? Journal of Archaeological Science37(10), 1-9.

Morgado, A. \&Baena,J. (2011). Experimentación, Arqueología experimental y experiencia del pasado en la Arqueología actual. En: Morgado A., Baena Preysler J. y García Gonzales D. (Eds.), La investigación experimental aplicada a la arqueología.Málaga:Ronda.

Ortega F.V. \&Marconetto, M.(2011). La explotación de los recursos combustibles:su uso y representación en la costa rionegrina a través de los restos antracológicos. En: F. Borello\& M. Cardillo (Eds.),Arqueología de Pescadores y Marisqueadores en Nordpatagonia. Descifrando un registro de más de 6000 años. Buenos Aires : EditorialDunken.

Pastor-Villegas, J., Rodríguez Meneses,J.M., Pastor-Valle, D.F.\& García García, M.(2007).Changes in commercial Wood charcoalbythermaltreatments. Journal of Analytical and Applied Pyrolysis 80(2), 507-514.

Pellegrin, J. (2011). Las experimentaciones aplicadas a la tecnología lítica. En: Morgado A., Baena Preysler J. y García Gonzales D. (Eds.), La investigación experimental aplicada a la arqueología. Málaga: Ronda.

Pérez de Micou, C. (1988).Paleoetnobotánica y territorios de explotación en asentamientos cazadores-recolectores. Precirculados de las Ponencias Científicas presentadas a los Simposiosdel IX Congreso Nacional de Arqueología Argentina. Instituto de Ciencias Antropológicas, Universidad de Buenos Aires, pp. 52-63.

Pérez de Micou, C. (1999). La recolección vegetal y la paleo-
etnobotánica.En: C. Aschero, A. Korstanje\& P. Vuoto (Eds.), En los tres reinos: Prácticas de recolección en el cono sur de América, pp. 27-37. Tucumán: Magna Publicaciones.

Perlès, C.(1977).Préhistoire du feu. París: Editorial Masson.

Piqué, I. \& Huerta, R. (1999).Producción y uso del combustible vegetal: unaevaluación arqueológica. Madrid: Universidad Autónomade Barcelona, Consejo Superior de Investigaciones Científicas.

Prior, J. \&Alvin, K.L. (1983).Structuralchangesoncharringwoods of Dichrostachys and Salixfrom southern Africa. International Association of Wood Anatomists Bulletin 4, 197-206.

Prior, J. \&Alvin, K.L. (1986).Structuralchanges n charringwoods of Dichrostachys and Salixfrom Southern Africa: theeffect of moisturecontent. IAWA Journal 7 (3), 243-249.

Prior, J. \&Gasson, P. (1993).AnatomicalchangesoncharringsixAfricanhardwoods. IAWA Journal 14, 77-86.

Py, V. \&Ancel, B. (2006).Archaeologicalexperiments in fire-setting: protocoland anthracologicalapproadi.En: CharroalAnn alysir:NeuiAnalyticalToolr and MethodiforArchaeology. PaperrfromtheTablr-Ronde heU in Barl2004. Oxford: Archaeopress (BAR International Series1483).

Rodriguez, M. F. (2003-2005). Aprovisionamiento y uso de recursos vegetales en la Patagonia meridional (República Argentina: Santa Cruz).Sitio arqueológico Cerro Casa de Piedra 5. Cuadernos del Instituto Nacional de Antropología y Pensamiento Latinoamericano 20, 339-355.

Scott, A., Cripps, J.,Collinson, M.\&Nichols, G.(2000).Thetaphonomy of charcoalfollowing a recenthearthlandfire and someimplicationsfortheinterpretation of fossilcharcoaldeposits. Palaeogeography, Palaecoclimatology, Palaeoecology164(1-4), 1-31.

Solari, M.E. (1993).L'homme et le bois en patagonie et terre de feuaucours des sixderniersmillénaires: Recherchesanthracologiquesau Chili et en Argentine. Tesis Doctoral,Université Montpellier II.

Solari, M.E. (2009). Análisis antracológico de una columna estratigráfica del Alero Cerro Castillo (Magallanes, Chile). Magallania37(1), 157-162.

Théry-Parisot, I. (1998).Économie du Combustible et Paléoécologie en contexteglaciaire et périglaciaire, Paléolithiquemoyen et supérieur du sud de la France. (Anthracologie, Expérimentation, Taphonomie). Tesis Doctoral,Université de Paris I Panthéon-Sorbonne.

Théry-Parisot, I.\&Costamagno, S. (2005).Propriétés combustibles des ossements: Donnéesexpérimentales et réflexionsarchéologiques sur leuremploidans les sitespaléolithiques. GalliaPréhistoire, 47, 235-254. 
Veblen, T. \&Ashton, D.H. (1982).Theregeneration status of Fitzroyacupressoides in the cordillera Pelada, Chile. BiolCons23, 141-61

Vidal, R. \&Premoli, A.(2004). Variación en Nothofagusantarctica (Forster) Oerst. (Nirre o Nire) En: Donoso C. L. Gallo,
A.Premoli\& R. Ipinza (Eds.) Variación intraespecifica en las especies arbóreas de los bosques templados de Chile y Argentina. Santiago: Editorial Universitaria.

Zicherman, J.B. (1981).Microstructure of woodchar. Wood Science and Technology 15, 237-249. 